\title{
SPARSE APPROXIMATION AND RECOVERY BY GREEDY ALGORITHMS IN BANACH SPACES
}

\author{
V. N. TEMLYAKOV \\ University of South Carolina, USA \\ Steklov Institute of Mathematics, Russia; \\ email: temlyakovusc@gmail.com
}

Received 5 June 2013; accepted 28 February 2014

\begin{abstract}
We study sparse approximation by greedy algorithms. We prove the Lebesgue-type inequalities for the weak Chebyshev greedy algorithm (WCGA), a generalization of the weak orthogonal matching pursuit to the case of a Banach space. The main novelty of these results is a Banach space setting instead of a Hilbert space setting. The results are proved for redundant dictionaries satisfying certain conditions. Then we apply these general results to the case of bases. In particular, we prove that the WCGA provides almost optimal sparse approximation for the trigonometric system in $L_{p}$, $2 \leqslant p<\infty$.
\end{abstract}

2010 Mathematics Subject Classification: primary 41A65; secondary 41A25, 41A46, 46B20.

\section{Introduction}

This paper is devoted to theoretical aspects of sparse approximation. The main motivation for the study of sparse approximation is that many real world signals can be well approximated by sparse ones. Sparse approximation automatically implies a need for nonlinear approximation, and in particular, for greedy approximation. We give a brief description of a sparse approximation problem in this section and present a detailed discussion of the results obtained and their relation to previous work in Section 5. In a general setting, we are working in a Banach space $X$ with a redundant system of elements $\mathcal{D}$ (dictionary $\mathcal{D})$. There is a solid justification for the importance of a Banach space setting in numerical analysis in general and in sparse approximation in particular (see, for instance, [18], Preface, and [14]). An element (function, or signal) $f \in X$ is

(c) The Author 2014. The online version of this article is published within an Open Access environment subject to the conditions of the Creative Commons Attribution licence <http://creativecommons.org/licenses/by/3.0/>. 
said to be $K$-sparse with respect to $\mathcal{D}$ if it has a representation $f=\sum_{i=1}^{K} x_{i} g_{i}$, $g_{i} \in \mathcal{D}, i=1, \ldots, K$. The set of all $K$-sparse elements is denoted by $\Sigma_{K}(\mathcal{D})$. For a given element $f_{0}$ we introduce the error of the best $m$-term approximation

$$
\sigma_{m}\left(f_{0}, \mathcal{D}\right):=\inf _{f \in \Sigma_{m}(\mathcal{D})}\left\|f_{0}-f\right\| .
$$

We are interested in the following fundamental problem of sparse approximation.

PROBLEM. How does one design a practical algorithm that builds sparse approximations comparable to the best $m$-term approximations?

We demonstrate in this paper that the weak Chebyshev greedy algorithm (WCGA) which we define shortly is a solution to the above problem. This paper is devoted to the Banach space setting. Let $X$ be a real Banach space with norm $\|\cdot\|:=\|\cdot\|_{X}$. We say that a set of elements (or functions) $\mathcal{D}$ from $X$ is a dictionary if each $g \in \mathcal{D}$ has norm one $(\|g\|=1)$, and the closure of $\operatorname{span} \mathcal{D}$ is $X$. For a nonzero element $g \in X$ we let $F_{g}$ denote a norming (peak) functional for $g$ :

$$
\left\|F_{g}\right\|_{X^{*}}=1, \quad F_{g}(g)=\|g\|_{X} .
$$

The existence of such a functional is guaranteed by the Hahn-Banach theorem.

Let $\tau:=\left\{t_{k}\right\}_{k=1}^{\infty}$ be a given weakness sequence of nonnegative numbers $t_{k} \leqslant 1$, $k=1, \ldots$ We define the weak Chebyshev greedy algorithm (WCGA) (see [16]) as a generalization for Banach spaces of the weak orthogonal matching pursuit (WOMP). In a Hilbert space the WCGA coincides with the WOMP. The WOMP is very popular in signal processing, and in particular, in compressed sensing. We study the WCGA in detail in this paper.

The weak Chebyshev greedy algorithm (WCGA). Let $f_{0}$ be given. Then for each $m \geqslant 1$ we have the following inductive definition.

(1) $\varphi_{m}:=\varphi_{m}^{c, \tau} \in \mathcal{D}$ is any element satisfying

$$
\left|F_{f_{m-1}}\left(\varphi_{m}\right)\right| \geqslant t_{m} \sup _{g \in \mathcal{D}}\left|F_{f_{m-1}}(g)\right| .
$$

(2) Define

$$
\Phi_{m}:=\Phi_{m}^{\tau}:=\operatorname{span}\left\{\varphi_{j}\right\}_{j=1}^{m},
$$

and define $G_{m}:=G_{m}^{c, \tau}$ to be the best approximant to $f_{0}$ from $\Phi_{m}$.

(3) Let

$$
f_{m}:=f_{m}^{c, \tau}:=f_{0}-G_{m} .
$$

In this paper we only consider the case where $t_{k}=t \in(0,1], k=1,2, \ldots$. 
The trigonometric system is a classical system that is known to be difficult to study. In this paper we study among other problems the problem of nonlinear sparse approximation with respect to it. Let $\mathcal{R} \mathcal{T}$ denote the real trigonometric system $1, \sin 2 \pi x, \cos 2 \pi x, \ldots$ on $[0,1]$ and let $\mathcal{R} \mathcal{T}_{p}$ to be its version normalized in $L_{p}([0,1])$. Denote as $\mathcal{R} \mathcal{T}_{p}^{d}:=\mathcal{R} \mathcal{T}_{p} \times \cdots \times \mathcal{R} \mathcal{T}_{p}$ the $d$-variate trigonometric system. We need to consider the real trigonometric system because the algorithm WCGA is well studied for the real Banach space. In order to illustrate the performance of the WCGA we discuss in this section the above mentioned problem for the trigonometric system. There is a natural algorithm, the thresholding greedy algorithm (TGA), that can be considered for the above problem. We give a definition of the TGA for a general basis $\Psi$. Let a Banach space $X$, with a normalized basis $\Psi=\left\{\psi_{k}\right\}_{k=1}^{\infty}$, be given. We consider the following greedy algorithm. For a given element $f \in X$ we consider the expansion

$$
f=\sum_{k=1}^{\infty} c_{k}(f) \psi_{k} .
$$

For an element $f \in X$ we say that a permutation $\rho$ of the positive integers is decreasing if

$$
\left|c_{k_{1}}(f)\right| \geqslant\left|c_{k_{2}}(f)\right| \geqslant \cdots,
$$

where $\rho(j)=k_{j}, j=1,2, \ldots$, and write $\rho \in D(f)$. If the inequalities are strict in (1.2), then $D(f)$ consists of only one permutation. We define the $m$ th greedy approximant of $f$, with regard to the basis $\Psi$ corresponding to a permutation $\rho \in D(f)$, by the formula

$$
G_{m}(f, \Psi):=G_{m}(f, \Psi, \rho):=\sum_{j=1}^{m} c_{k_{j}}(f) \psi_{k_{j}} .
$$

The following Lebesgue-type inequality was proved in [15].

THEOREM 1.1. For each $f \in L_{p}\left([0,1]^{d}\right)$ we have

$$
\left\|f-G_{m}\left(f, \mathcal{R} \mathcal{T}_{p}^{d}\right)\right\|_{p} \leqslant C(d) m^{h(p)} \sigma_{m}\left(f, \mathcal{R} \mathcal{T}_{p}^{d}\right)_{p}, \quad 1 \leqslant p \leqslant \infty,
$$

where $h(p):=|1 / 2-1 / p|$.

It was also proved in [15] that the above inequality is sharp.

REMARK 1.1. There is a positive absolute constant $C$ such that for each $m$ and $1 \leqslant p \leqslant \infty$ there exists a function $f \neq 0$ with the property

$$
\left\|G_{m}\left(f, \mathcal{R} \mathcal{T}_{p}\right)\right\|_{p} \geqslant C m^{h(p)}\|f\|_{p} .
$$


Remark 1.1 shows that the TGA does not work well for the trigonometric system in $L_{p}, p \neq 2$. This leads to a natural attempt to consider some other algorithms that may have some advantages over the TGA in the case of the trigonometric system. In this paper we discuss the performance of the weak Chebyshev greedy algorithm (WCGA) with respect to the trigonometric system. We prove here the following Lebesgue-type inequality for the WCGA (see Example 2 in Section 4).

THEOREM 1.2. Let $\mathcal{D}$ be the real $d$-variate trigonometric system normalized in $L_{p}, 2 \leqslant p<\infty$. Then for any $f_{0} \in L_{p}$ the WCGA with weakness parameter $t$ gives

$$
\left\|f_{C(t, p, d) m \ln (m+1)}\right\|_{p} \leqslant C \sigma_{m}\left(f_{0}, \mathcal{D}\right)_{p} .
$$

The Open Problem 7.1 (p. 91) from [17] asks if (1.4) holds without an extra $\ln (m+1)$ factor. Theorem 1.2 is the first result on the Lebesgue-type inequalities for the WCGA with respect to the trigonometric system. It provides progress in solving the above mentioned open problem, but the problem is still open.

Theorem 1.2 shows that the WCGA is very well designed for the trigonometric system. We show in Example 1 of Section 4 that an analog of (1.4) holds for uniformly bounded orthogonal systems. We note that it is known (see [18]) that the TGA is very well designed for bases $L_{p}$-equivalent to the Haar basis, $1<p<$ $\infty$. We discuss the performance of the WCGA in more detail in Section 5.

The proof of Theorem 1.2 uses a technique developed for proving the Lebesgue-type inequalities for redundant dictionaries with special properties. We present these results in Sections 2 and 3. These results are an extension of earlier results from [10]. In Section 4 we test the power of general results from Section 2 on specific dictionaries, namely, on bases. Section 4 provides a number of examples, including the trigonometric system, were the technique from Sections 2 and 3 can be successfully applied. In particular, results from Section 4 demonstrate that the general technique from Sections 2 and 3 provides almost optimal $m$-term approximation results for uniformly bounded orthogonal systems (see Example 1). Example 7 shows that an extra assumption that a uniformly bounded orthogonal system $\Psi$ is a quasigreedy basis allows us to improve inequality (1.4):

$$
\left\|f_{C(t, p, \Psi) m \ln \ln (m+3)}\right\|_{p} \leqslant C \sigma_{m}\left(f_{0}, \Psi\right)_{p} .
$$

\section{Lebesgue-type inequalities; general results}

A very important advantage of the WCGA is its convergence and rate of convergence properties. The WCGA is well defined for all $\mathrm{m}$. Moreover, it is 
known (see [16] and [18]) that the WCGA with $\tau=\{t\}$ converges for all $f_{0}$ in all uniformly smooth Banach spaces with respect to any dictionary. That is, when $X$ is a real Banach space and the modulus of smoothness of $X$ is defined as follows:

$$
\rho(u):=\frac{1}{2} \sup _{x, y ;\|x\|=\|y\|=1}|\|x+u y\|+\|x-u y\|-2|,
$$

then the uniformly smooth Banach space is the one with $\rho(u) / u \rightarrow 0$ when $u \rightarrow 0$.

We discuss here the Lebesgue-type inequalities for the WCGA with $\tau=\{t\}$, $t \in(0,1]$. For notational convenience we consider here a countable dictionary $\mathcal{D}=\left\{g_{i}\right\}_{i=1}^{\infty}$. The following assumptions $\mathbf{A 1}$ and $\mathbf{A 2}$ were used in [10]. For a given $f_{0}$ let the sparse element (or signal)

$$
f:=f^{\epsilon}=\sum_{i \in T} x_{i} g_{i}
$$

be such that $\left\|f_{0}-f^{\epsilon}\right\| \leqslant \epsilon$ and $|T|=K$. For $A \subset T$ define

$$
f_{A}:=f_{A}^{\epsilon}:=\sum_{i \in A} x_{i} g_{i}
$$

A1. We say that $f=\sum_{i \in T} x_{i} g_{i}$ satisfies the Nikol'skii-type $\ell_{1} X$ inequality with parameter $r$ if

$$
\sum_{i \in A}\left|x_{i}\right| \leqslant C_{1}|A|^{r}\left\|f_{A}\right\|, \quad A \subset T, \quad r \geqslant 1 / 2
$$

We say that a dictionary $\mathcal{D}$ has the Nikol'skii-type $\ell_{1} X$ property with parameters $K, r$ if any $K$-sparse element satisfies the Nikol'skii-type $\ell_{1} X$ inequality with parameter $r$.

A2. We say that $f=\sum_{i \in T} x_{i} g_{i}$ has the incoherence property with parameters $D$ and $U$ if for any $A \subset T$ and any $\Lambda$ such that $A \cap \Lambda=\emptyset,|A|+|\Lambda| \leqslant D$ we have for any $\left\{c_{i}\right\}$,

$$
\left\|f_{A}-\sum_{i \in \Lambda} c_{i} g_{i}\right\| \geqslant U^{-1}\left\|f_{A}\right\| .
$$

We say that a dictionary $\mathcal{D}$ is $(K, D)$-unconditional with a constant $U$ if for any $f=\sum_{i \in T} x_{i} g_{i}$ with $|T| \leqslant K$, inequality (2.3) holds.

The term unconditional in $\mathbf{A 2}$ is justified by the following remark. The above definition of a $(K, D)$-unconditional dictionary is equivalent to the following definition. Let $\mathcal{D}$ be such that any subsystem of $D$ distinct elements $e_{1}, \ldots, e_{D}$ 
from $\mathcal{D}$ is linearly independent and for any $A \subset[1, D]$ with $|A| \leqslant K$ and any coefficients $\left\{c_{i}\right\}$ we have

$$
\left\|\sum_{i \in A} c_{i} e_{i}\right\| \leqslant U\left\|\sum_{i=1}^{D} c_{i} e_{i}\right\| .
$$

It is convenient for us to use the following assumption $\mathbf{A 3}$ which is a corollary of assumptions A1 and $\mathbf{A 2}$.

A3. We say that $f=\sum_{i \in T} x_{i} g_{i}$ has the $\ell_{1}$ incoherence property with parameters $D, V$, and $r$ if for any $A \subset T$ and any $\Lambda$ such that $A \cap \Lambda=\emptyset,|A|+|\Lambda| \leqslant D$ we have for any $\left\{c_{i}\right\}$,

$$
\sum_{i \in A}\left|x_{i}\right| \leqslant V|A|^{r}\left\|f_{A}-\sum_{i \in \Lambda} c_{i} g_{i}\right\| .
$$

A dictionary $\mathcal{D}$ has the $\ell_{1}$ incoherence property with parameters $K, D, V$, and $r$ if for any $A \subset B,|A| \leqslant K,|B| \leqslant D$ we have for any $\left\{c_{i}\right\}_{i \in B}$,

$$
\sum_{i \in A}\left|c_{i}\right| \leqslant V|A|^{r}\left\|\sum_{i \in B} c_{i} g_{i}\right\| .
$$

It is clear that $\mathbf{A 1}$ and $\mathbf{A 2}$ imply $\mathbf{A} \mathbf{3}$ with $V=C_{1} U$. Also, $\mathbf{A 3}$ implies $\mathbf{A 1}$ with $C_{1}=V$ and $\mathbf{A} 2$ with $U=V K^{r}$. Obviously, we can restrict ourselves to $r \leqslant 1$.

We now proceed to the main results of this paper on the WCGA with respect to redundant dictionaries. The following theorem, Theorem 2.1, was proved in [10] for the case $q=2$.

THEOREM 2.1. Let $X$ be a Banach space with $\rho(u) \leqslant \gamma u^{q}, 1<q \leqslant 2$. Suppose $K$-sparse $f^{\epsilon}$ satisfies A1, A2 and $\left\|f_{0}-f^{\epsilon}\right\| \leqslant \epsilon$. Then the WCGA with weakness parameter $t$ applied to $f_{0}$ provides

$$
\left\|f_{C\left(t, \gamma, C_{1}\right) U^{q^{\prime}} \ln (U+1) K^{r q^{\prime}}}\right\| \leqslant C \epsilon \quad \text { for } K+C\left(t, \gamma, C_{1}\right) U^{q^{\prime}} \ln (U+1) K^{r q^{\prime}} \leqslant D
$$

with an absolute constant $C$.

It was pointed out in [10] that Theorem 2.1 provides a corollary for Hilbert spaces that gives sufficient conditions somewhat weaker than the known RIP conditions on $\mathcal{D}$ for the Lebesgue-type inequality to hold. We formulate the corresponding definitions and results. Let $\mathcal{D}$ be the Riesz dictionary with depth $D$ and parameter $\delta \in(0,1)$. This class of dictionaries is a generalization of the class of classical Riesz bases. We give a definition for in a general Hilbert space (see [18], p. 306). 
Definition 2.1. A dictionary $\mathcal{D}$ is called the Riesz dictionary with depth $D$ and parameter $\delta \in(0,1)$ if, for any $D$ distinct elements $e_{1}, \ldots, e_{D}$ of the dictionary and any coefficients $a=\left(a_{1}, \ldots, a_{D}\right)$, we have

$$
(1-\delta)\|a\|_{2}^{2} \leqslant\left\|\sum_{i=1}^{D} a_{i} e_{i}\right\|^{2} \leqslant(1+\delta)\|a\|_{2}^{2} .
$$

We denote the class of Riesz dictionaries with depth $D$ and parameter $\delta \in(0,1)$ by $R(D, \delta)$.

The term Riesz dictionary with depth $D$ and parameter $\delta \in(0,1)$ is another name for a dictionary satisfying the restricted isometry property (RIP) with parameters $D$ and $\delta$. The following simple lemma holds.

Lemma 2.1. Let $\mathcal{D} \in R(D, \delta)$ and let $e_{j} \in \mathcal{D}, j=1, \ldots, s$. For $f=\sum_{i=1}^{s} a_{i} e_{i}$ and $A \subset\{1, \ldots, s\}$, define

$$
S_{A}(f):=\sum_{i \in A} a_{i} e_{i}
$$

If $s \leqslant D$ then

$$
\left\|S_{A}(f)\right\|^{2} \leqslant(1+\delta)(1-\delta)^{-1}\|f\|^{2} .
$$

Lemma 2.1 implies that if $\mathcal{D} \in R(D, \delta)$ then it is $(D, D)$-unconditional with a constant $U=(1+\delta)^{1 / 2}(1-\delta)^{-1 / 2}$.

Theorem 2.2. Let $X$ be a Hilbert space. Suppose $K$-sparse $f^{\epsilon}$ satisfies $\mathbf{A 2}$ and $\left\|f_{0}-f^{\epsilon}\right\| \leqslant \epsilon$. Then the WOMP with weakness parameter $t$ applied to $f_{0}$ provides

$$
\left\|f_{C(t, U) K}\right\| \leqslant C \epsilon \quad \text { for } K+C(t, U) K \leqslant D
$$

with an absolute constant $C$.

Theorem 2.2 implies the following corollaries.

Corollary 2.1. Let $X$ be a Hilbert space. Suppose any $K$-sparse $f$ satisfies A2. Then the WOMP with weakness parameter $t$ applied to $f_{0}$ provides

$$
\left\|f_{C(t, U) K}\right\| \leqslant C \sigma_{K}\left(f_{0}, \mathcal{D}\right) \quad \text { for } K+C(t, U) K \leqslant D
$$

with an absolute constant $C$. 
Corollary 2.2. Let $X$ be a Hilbert space. Suppose $\mathcal{D} \in R(D, \delta)$. Then the WOMP with weakness parameter $t$ applied to $f_{0}$ provides

$$
\left\|f_{C(t, \delta) K}\right\| \leqslant C \sigma_{K}\left(f_{0}, \mathcal{D}\right) \quad \text { for } K+C(t, \delta) K \leqslant D
$$

with an absolute constant $C$.

We emphasized in [10] that in Theorem 2.1 we impose our conditions on an individual function $f^{\epsilon}$. It may happen that the dictionary does not have the Nikol'skii $\ell_{1} X$ property and $(K, D)$-unconditionality, but the given $f_{0}$ can be approximated by $f^{\epsilon}$ which does satisfy assumptions A1 and A2. Even in the case of a Hilbert space the above results from [10] add something new to the study based on the RIP property of a dictionary. First of all, Theorem 2.2 shows that it is sufficient to impose assumption $\mathbf{A} \mathbf{2}$ on $f^{\epsilon}$ to obtain exact recovery and the Lebesgue-type inequality results. Second, Corollary 2.1 shows that the condition A2, which is weaker than the RIP condition, is sufficient for exact recovery and the Lebesgue-type inequality results. Third, Corollary 2.2 shows that even if we impose our assumptions in terms of RIP, we do not need to assume that $\delta<\delta_{0}$. In fact, the result works for all $\delta<1$ with parameters depending on $\delta$.

Theorem 2.1 follows from the combination of Theorems 2.3 and 2.4. For the case $q=2$, these theorems were proved in [10].

THEOREM 2.3. Let $X$ be a Banach space with $\rho(u) \leqslant \gamma u^{q}, 1<q \leqslant 2$. Suppose for a given $f_{0}$ we have $\left\|f_{0}-f^{\epsilon}\right\| \leqslant \epsilon$ with $K$-sparse $f:=f^{\epsilon}$ satisfying A3. Then for any $k \geqslant 0$ we have for $K+m \leqslant D$,

$$
\left\|f_{m}\right\| \leqslant\left\|f_{k}\right\| \exp \left(-\frac{c_{1}(m-k)}{K^{r q^{\prime}}}\right)+2 \epsilon, \quad q^{\prime}:=\frac{q}{q-1},
$$

where $c_{1}:=\left(t^{q^{\prime}}\right) /\left(2(16 \gamma)^{1 /(q-1)} V^{q^{\prime}}\right)$.

In all of the theorems that follow, we assume that $r q^{\prime} \geqslant 1$.

THEOREM 2.4. Let $X$ be a Banach space with $\rho(u) \leqslant \gamma u^{q}, 1<q \leqslant 2$. Suppose $K$-sparse $f^{\epsilon}$ satisfies A1, A2 and $\left\|f_{0}-f^{\epsilon}\right\| \leqslant \epsilon$. Then the WCGA with weakness parameter $t$ applied to $f_{0}$ provides

$$
\left\|f_{C^{\prime} U^{q^{\prime}} \ln (U+1) K^{r q^{\prime}}}\right\| \leqslant C U \epsilon \quad \text { for } K+C^{\prime} U^{q^{\prime}} \ln (U+1) K^{r q^{\prime}} \leqslant D
$$

with an absolute constant $C$ and $C^{\prime}=C_{2}(q) \gamma^{(1 / q-1)} C_{1}^{q^{\prime}} t^{-q^{\prime}}$.

We formulate an immediate corollary of Theorem 2.4 with $\epsilon=0$. 
Corollary 2.3. Let $X$ be a Banach space with $\rho(u) \leqslant \gamma u^{q}$. Suppose $K$ sparse $f$ satisfies $\mathbf{A 1}, \mathbf{A 2}$. Then the WCGA with weakness parameter $t$ applied to $f$ recovers it exactly after $C^{\prime} U^{q^{\prime}} \ln (U+1) K^{r q^{\prime}}$ iterations under condition $K+C^{\prime} U^{q^{\prime}} \ln (U+1) K^{r q^{\prime}} \leqslant D$.

We formulate versions of Theorem 2.4 with assumptions A1, A2 replaced by a single assumption A3 and replaced by two assumptions A2 and A3. The corresponding modifications in the proofs go as in the proof of Theorem 2.3.

THEOREM 2.5. Let $X$ be a Banach space with $\rho(u) \leqslant \gamma u^{q}, 1<q \leqslant 2$. Suppose $K$-sparse $f^{\epsilon}$ satisfies $\mathbf{A} 3$ and $\left\|f_{0}-f^{\epsilon}\right\| \leqslant \epsilon$. Then the WCGA with weakness parameter $t$ applied to $f_{0}$ provides

$$
\left\|f_{C(t, \gamma, q) V^{q^{\prime}} \ln (V K) K^{r q^{\prime}}}\right\| \leqslant C V K^{r} \epsilon \text { for } K+C(t, \gamma, q) V^{q^{\prime}} \ln (V K) K^{r q^{\prime}} \leqslant D
$$

with an absolute constant $C$ and $C(t, \gamma, q)=C_{2}(q) \gamma^{1 /(q-1)} t^{-q^{\prime}}$.

THEOREM 2.6. Let $X$ be a Banach space with $\rho(u) \leqslant \gamma u^{q}, 1<q \leqslant 2$. Suppose $K$-sparse $f^{\epsilon}$ satisfies $\mathbf{A 2}$, A3 and $\left\|f_{0}-f^{\epsilon}\right\| \leqslant \epsilon$. Then the WCGA with weakness parameter $t$ applied to $f_{0}$ provides

$$
\left\|f_{C(t, \gamma, q) V^{q^{\prime}} \ln (U+1) K^{r q^{\prime}}}\right\| \leqslant C U \epsilon \text { for } K+C(t, \gamma, q) V^{q^{\prime}} \ln (U+1) K^{r q^{\prime}} \leqslant D
$$

with an absolute constant $C$ and $C(t, \gamma, q)=C_{2}(q) \gamma^{1 /(q-1)} t^{-q^{\prime}}$.

Theorems 2.5 and 2.3 imply the following analog of Theorem 2.1.

THEOREM 2.7. Let $X$ be a Banach space with $\rho(u) \leqslant \gamma u^{q}, 1<q \leqslant 2$. Suppose $K$-sparse $f^{\epsilon}$ satisfies $\mathbf{A} \mathbf{3}$ and $\left\|f_{0}-f^{\epsilon}\right\| \leqslant \epsilon$. Then the WCGA with weakness parameter $t$ applied to $f_{0}$ provides

$$
\left\|f_{C(t, \gamma, q) V^{q^{\prime}} \ln (V K) K^{r q^{\prime}}}\right\| \leqslant C \epsilon \text { for } K+C(t, \gamma, q) V^{q^{\prime}} \ln (V K) K^{r q^{\prime}} \leqslant D
$$

with an absolute constant $C$ and $C(t, \gamma, q)=C_{2}(q) \gamma^{1 /(q-1)} t^{-q^{\prime}}$.

The following version of Theorems 2.1 and 2.7 is also useful in applications. It follows from Theorems 2.6 and 2.3.

Theorem 2.8. Let $X$ be a Banach space with $\rho(u) \leqslant \gamma u^{q}, 1<q \leqslant 2$. Suppose $K$-sparse $f^{\epsilon}$ satisfies $\mathbf{A 2}$, A3 and $\left\|f_{0}-f^{\epsilon}\right\| \leqslant \epsilon$. Then the WCGA with weakness parameter $t$ applied to $f_{0}$ provides

$$
\left\|f_{C(t, \gamma, q) V^{q^{\prime}} \ln (U+1) K^{r q^{\prime}}}\right\| \leqslant C \epsilon \text { for } K+C(t, \gamma, q) V^{q^{\prime}} \ln (U+1) K^{r q^{\prime}} \leqslant D
$$

with an absolute constant $C$ and $C(t, \gamma, q)=C_{2}(q) \gamma^{1 /(q-1)} t^{-q^{\prime}}$. 


\section{Proofs}

We begin with a proof of Theorem 2.3.

Proof. Let

$$
f:=f^{\epsilon}=\sum_{i \in T} x_{i} g_{i}, \quad|T|=K, \quad g_{i} \in \mathcal{D} .
$$

Denote by $T^{m}$ the set of indices of $g_{j} \in D$ picked by the WCGA after $m$ iterations, $\Gamma^{m}:=T \backslash T^{m}$. Denote by $A_{1}(\mathcal{D})$ the closure in $X$ of the convex hull of the symmetrized dictionary $\mathcal{D}^{ \pm}:=\{ \pm g, g \in D\}$. We will bound $\left\|f_{m}\right\|$ from above. Assume that $\left\|f_{m-1}\right\| \geqslant \epsilon$. Let $m>k$. We bound from below:

$$
S_{m}:=\sup _{\phi \in A_{1}(\mathcal{D})}\left|F_{f_{m-1}}(\phi)\right| .
$$

Define $A_{m}:=\Gamma^{m-1}$. Then

$$
S_{m} \geqslant F_{f_{m-1}}\left(f_{A_{m}} /\left\|f_{A_{m}}\right\|_{1}\right),
$$

where $\left\|f_{A}\right\|_{1}:=\sum_{i \in A}\left|x_{i}\right|$. Next, by Lemma 6.9, p. 342, from [18] we obtain

$$
F_{f_{m-1}}\left(f_{A_{m}}\right)=F_{f_{m-1}}\left(f^{\epsilon}\right) \geqslant\left\|f_{m-1}\right\|-\epsilon .
$$

Thus

$$
S_{m} \geqslant\left\|f_{A_{m}}\right\|_{1}^{-1}\left(\left\|f_{m-1}\right\|-\epsilon\right) .
$$

From the definition of the modulus of smoothness we have for any $\lambda$,

$$
\left\|f_{m-1}-\lambda \varphi_{m}\right\|+\left\|f_{m-1}+\lambda \varphi_{m}\right\| \leqslant 2\left\|f_{m-1}\right\|\left(1+\rho\left(\frac{\lambda}{\left\|f_{m-1}\right\|}\right)\right),
$$

and by (1), from the definition of the WCGA and Lemma 6.10 from [18], p. 343, we get

$$
\begin{aligned}
\left|F_{f_{m-1}}\left(\varphi_{m}\right)\right| & \geqslant t \sup _{g \in \mathcal{D}}\left|F_{f_{m-1}}(g)\right| \\
& =t \sup _{\phi \in A_{1}(\mathcal{D})}\left|F_{f_{m-1}}(\phi)\right|=t S_{m} .
\end{aligned}
$$

Then either $F_{f_{m-1}}\left(\varphi_{m}\right) \geqslant t S_{m}$ or $F_{f_{m-1}}\left(-\varphi_{m}\right) \geqslant t S_{m}$. The two cases are treated in the same way. We demonstrate the case $F_{f_{m-1}}\left(\varphi_{m}\right) \geqslant t S_{m}$. We have for $\lambda \geqslant 0$,

$$
\left\|f_{m-1}+\lambda \varphi_{m}\right\| \geqslant F_{f_{m-1}}\left(f_{m-1}+\lambda \varphi_{m}\right) \geqslant\left\|f_{m-1}\right\|+\lambda t S_{m}
$$

From here and from (3.2) we obtain

$$
\left\|f_{m}\right\| \leqslant\left\|f_{m-1}-\lambda \varphi_{m}\right\| \leqslant\left\|f_{m-1}\right\|+\inf _{\lambda \geqslant 0}\left(-\lambda t S_{m}+2\left\|f_{m-1}\right\| \rho\left(\lambda /\left\|f_{m-1}\right\|\right)\right) .
$$


We discuss here the case $\rho(u) \leqslant \gamma u^{q}$. Using (3.1) we get

$$
\left\|f_{m}\right\| \leqslant\left\|f_{m-1}\right\|\left(1-\frac{\lambda t}{\left\|f_{A_{m}}\right\|_{1}}+2 \gamma \frac{\lambda^{q}}{\left\|f_{m-1}\right\|^{q}}\right)+\frac{\epsilon \lambda t}{\left\|f_{A_{m}}\right\|_{1}} .
$$

Let $\lambda_{1}$ be a solution of

$$
\frac{\lambda t}{2\left\|f_{A_{m}}\right\|_{1}}=2 \gamma \frac{\lambda^{q}}{\left\|f_{m-1}\right\|^{q}}, \quad \lambda_{1}=\left(\frac{t\left\|f_{m-1}\right\|^{q}}{4 \gamma\left\|f_{A_{m}}\right\|_{1}}\right)^{\frac{1}{q-1}} .
$$

Our assumption (2.4) gives

$$
\begin{aligned}
\left\|f_{A_{m}}\right\|_{1} & =\left\|\left(f^{\epsilon}-G_{m-1}\right)_{A_{m}}\right\|_{1} \leqslant V K^{r}\left\|f^{\epsilon}-G_{m-1}\right\| \\
& \leqslant V K^{r}\left(\left\|f_{0}-G_{m-1}\right\|+\left\|f_{0}-f^{\epsilon}\right\|\right) \leqslant V K^{r}\left(\left\|f_{m-1}\right\|+\epsilon\right) .
\end{aligned}
$$

Specify

$$
\lambda=\left(\frac{t\left\|f_{A_{m}}\right\|_{1}^{q-1}}{16 \gamma\left(V K^{r}\right)^{q}}\right)^{\frac{1}{q-1}} .
$$

Then, using $\left\|f_{m-1}\right\| \geqslant \epsilon$ we get

$$
\left(\frac{\lambda}{\lambda_{1}}\right)^{q-1}=\frac{\left\|f_{A_{m}}\right\|_{1}^{q}}{4\left\|f_{m-1}\right\|^{q}\left(V K^{r}\right)^{q}} \leqslant 1
$$

and obtain

$$
\left\|f_{m}\right\| \leqslant\left\|f_{m-1}\right\|\left(1-\frac{t^{q^{\prime}}}{2(16 \gamma)^{\frac{1}{q-1}}\left(V K^{r}\right)^{q^{\prime}}}\right)+\frac{\epsilon t^{q^{\prime}}}{(16 \gamma)^{\frac{1}{q-1}}\left(V K^{r}\right)^{q^{\prime}}} .
$$

Define $c_{1}:=\left(t^{q^{\prime}}\right) /\left(2(16 \gamma)^{1 /(q-1)} V^{q^{\prime}}\right)$. Then

$$
\left\|f_{m}\right\| \leqslant\left\|f_{k}\right\| \exp \left(-\frac{c_{1}(m-k)}{K^{r q^{\prime}}}\right)+2 \epsilon .
$$

We proceed to a proof of Theorem 2.4. Modifications of this proof which are in the style of the above proof of Theorem 2.3 give Theorems 2.5 and 2.6.

Proof. We use the above notation $T^{m}$ and $\Gamma^{m}:=T \backslash T^{m}$. Let $k \geqslant 0$ be fixed. Suppose that

$$
2^{n-1}<\left|\Gamma^{k}\right| \leqslant 2^{n} .
$$

For $j=1,2, \ldots, n, n+1$ consider the following pairs of sets $A_{j}, B_{j}: A_{n+1}=\Gamma^{k}$, $B_{n+1}=\emptyset$; for $j \leqslant n, A_{j}:=\Gamma^{k} \backslash B_{j}$ with $B_{j} \subset \Gamma^{k}$ is such that $\left|B_{j}\right| \geqslant\left|\Gamma^{k}\right|-2^{j-1}$ and for any set $J \subset \Gamma^{k}$ with $|J| \geqslant\left|\Gamma^{k}\right|-2^{j-1}$ we have

$$
\left\|f_{B_{j}}\right\| \leqslant\left\|f_{J}\right\| \text {. }
$$


We note that this implies that if for some $Q \subset \Gamma^{k}$ we have

$$
\left\|f_{Q}\right\|<\left\|f_{B_{j}}\right\| \quad \text { then }|Q|<\left|\Gamma^{k}\right|-2^{j-1} \text {. }
$$

For a given $b>1$, to be specified later, denote by $L$ the index such that $\left(B_{0}:=\Gamma^{k}\right)$

$$
\begin{aligned}
& \left\|f_{B_{0}}\right\|<b\left\|f_{B_{1}}\right\|, \\
& \left\|f_{B_{1}}\right\|<b\left\|f_{B_{2}}\right\|, \\
& \ldots \\
& \left\|f_{B_{L-2}}\right\|<b\left\|f_{B_{L-1}}\right\|, \\
& \left\|f_{B_{L-1}}\right\| \geqslant b\left\|f_{B_{L}}\right\| .
\end{aligned}
$$

Then

$$
\left\|f_{B_{j}}\right\| \leqslant b^{L-1-j}\left\|f_{B_{L-1}}\right\|, \quad j=1,2, \ldots, L .
$$

We now proceed to a general step. Let $m>k$ and let $A, B \subset \Gamma^{k}$ be such that $A=\Gamma^{k} \backslash B$. As above we bound $S_{m}$ from below. It is clear that $S_{m} \geqslant 0$. Define $A_{m}:=A \cap \Gamma^{m-1}$. Then

$$
S_{m} \geqslant F_{f_{m-1}}\left(f_{A_{m}} /\left\|f_{A_{m}}\right\|_{1}\right)
$$

Next,

$$
F_{f_{m-1}}\left(f_{A_{m}}\right)=F_{f_{m-1}}\left(f_{A_{m}}+f_{B}-f_{B}\right) .
$$

Then $f_{A_{m}}+f_{B}=f^{\epsilon}-f_{\Lambda}$ with $F_{f_{m-1}}\left(f_{\Lambda}\right)=0$. Moreover, it is easy to see that $F_{f_{m-1}}\left(f^{\epsilon}\right) \geqslant\left\|f_{m-1}\right\|-\epsilon$. Therefore,

$$
F_{f_{m-1}}\left(f_{A_{m}}+f_{B}-f_{B}\right) \geqslant\left\|f_{m-1}\right\|-\epsilon-\left\|f_{B}\right\| .
$$

Thus

$$
S_{m} \geqslant\left\|f_{A_{m}}\right\|_{1}^{-1} \max \left(0,\left\|f_{m-1}\right\|-\epsilon-\left\|f_{B}\right\|\right) .
$$

By (2.2) we get

$$
\left\|f_{A_{m}}\right\|_{1} \leqslant C_{1}\left|A_{m}\right|^{r}\left\|f_{A_{m}}\right\| \leqslant C_{1}|A|^{r}\left\|f_{A_{m}}\right\|
$$

Then

$$
S_{m} \geqslant \frac{\left\|f_{m-1}\right\|-\left\|f_{B}\right\|-\epsilon}{C_{1}|A|^{r}\left\|f_{A_{m}}\right\|} .
$$

From the definition of the modulus of smoothness we have for any $\lambda$,

$$
\left\|f_{m-1}-\lambda \varphi_{m}\right\|+\left\|f_{m-1}+\lambda \varphi_{m}\right\| \leqslant 2\left\|f_{m-1}\right\|\left(1+\rho\left(\frac{\lambda}{\left\|f_{m-1}\right\|}\right)\right)
$$


and by (1) from the definition of the WCGA and Lemma 6.10 from [18], p. 343, we get

$$
\begin{aligned}
\left|F_{f_{m-1}}\left(\varphi_{m}\right)\right| & \geqslant t \sup _{g \in \mathcal{D}}\left|F_{f_{m-1}}(g)\right| \\
& =t \sup _{\phi \in A_{1}(\mathcal{D})}\left|F_{f_{m-1}}(\phi)\right| .
\end{aligned}
$$

From here we obtain

$$
\left\|f_{m}\right\| \leqslant\left\|f_{m-1}\right\|+\inf _{\lambda \geqslant 0}\left(-\lambda t S_{m}+2\left\|f_{m-1}\right\| \rho\left(\lambda /\left\|f_{m-1}\right\|\right)\right) .
$$

We discuss here the case $\rho(u) \leqslant \gamma u^{q}$. Using (3.5) we get

$$
\left\|f_{m}\right\| \leqslant\left\|f_{m-1}\right\|\left(1-\frac{\lambda t}{C_{1}|A|^{r}\left\|f_{A_{m}}\right\|}+2 \gamma \frac{\lambda^{q}}{\left\|f_{m-1}\right\|^{q}}\right)+\frac{\lambda t\left(\left\|f_{B}\right\|+\epsilon\right)}{C_{1}|A|^{r}\left\|f_{A_{m}}\right\|} .
$$

Let $\lambda_{1}$ be a solution of

$$
\frac{\lambda t}{2 C_{1}|A|^{r}\left\|f_{A_{m}}\right\|}=2 \gamma \frac{\lambda^{q}}{\left\|f_{m-1}\right\|^{q}}, \quad \lambda_{1}=\left(\frac{t\left\|f_{m-1}\right\|^{q}}{4 \gamma C_{1}|A|^{r}\left\|f_{A_{m}}\right\|}\right)^{\frac{1}{q-1}} .
$$

Our assumption (2.3) gives

$$
\left\|f_{A_{m}}\right\| \leqslant U\left(\left\|f_{m-1}\right\|+\epsilon\right)
$$

Specify

$$
\lambda=\left(\frac{t\left\|f_{A_{m}}\right\|^{q-1}}{16 \gamma C_{1}|A|^{r} U^{q}}\right)^{\frac{1}{q-1}} .
$$

Then $\lambda \leqslant \lambda_{1}$ and we obtain

$$
\left\|f_{m}\right\| \leqslant\left\|f_{m-1}\right\|\left(1-\frac{t^{q^{\prime}}}{2(16 \gamma)^{\frac{1}{q-1}}\left(C_{1} U|A|^{r}\right)^{q^{\prime}}}\right)+\frac{t^{q^{\prime}}\left(\left\|f_{B}\right\|+\epsilon\right)}{(16 \gamma)^{\frac{1}{q-1}}\left(C_{1}|A|^{r} U\right)^{q^{\prime}}} .
$$

Define $c_{1}:=\left(t^{q^{\prime}}\right) /\left(2(16 \gamma)^{1 /(q-1)}\left(C_{1} U\right)^{q^{\prime}}\right)$. This implies for $m_{2}>m_{1} \geqslant k$,

$$
\left\|f_{m_{2}}\right\| \leqslant\left\|f_{m_{1}}\right\|\left(1-c_{1} /|A|^{r q^{\prime}}\right)^{m_{2}-m_{1}}+\frac{2 c_{1}\left(m_{2}-m_{1}\right)}{|A|^{2 r}}\left(\left\|f_{B}\right\|+\epsilon\right) .
$$

Define $m_{0}:=k$ and, inductively,

$$
m_{j}=m_{j-1}+\beta\left|A_{j}\right|^{r q^{\prime}}, \quad j=1, \ldots, n .
$$

At iterations from $m_{j-1}+1$ to $m_{j}$ we use $A=A_{j}$ and obtain from (3.6),

$$
\left\|f_{m_{j}}\right\| \leqslant\left\|f_{m_{j-1}}\right\| e^{-c_{1} \beta}+2\left(\left\|f_{B_{j}}\right\|+\epsilon\right)
$$


We continue it up to $j=L$. Define $\eta:=e^{-c_{1} \beta}$. Then

$$
\left\|f_{m_{L}}\right\| \leqslant\left\|f_{k}\right\| \eta^{L}+2 \sum_{j=1}^{L}\left(\left\|f_{B_{j}}\right\|+\epsilon\right) \eta^{L-j} .
$$

We bound the $\left\|f_{k}\right\|$. It follows from the definition of $f_{k}$ that $\left\|f_{k}\right\|$ is the error of best approximation of $f_{0}$ by the subspace $\Phi_{k}$. Representing $f_{0}=f+f_{0}-f$ we see that $\left\|f_{k}\right\|$ is not greater than the error of best approximation of $f$ by the subspace $\Phi_{k}$ plus $\left\|f_{0}-f\right\|$. This implies that $\left\|f_{k}\right\| \leqslant\left\|f_{B_{0}}\right\|+\epsilon$. Therefore we continue:

$$
\begin{gathered}
\leqslant\left(\left\|f_{B_{0}}\right\|+\epsilon\right) \eta^{L}+2 \sum_{j=1}^{L}\left(\left\|f_{B_{L-1}}\right\|(\eta b)^{L-j} b^{-1}+\epsilon \eta^{L-j}\right) \\
\leqslant b^{-1}\left\|f_{B_{L-1}}\right\|\left((\eta b)^{L}+2 \sum_{j=1}^{L}(\eta b)^{L-j}\right)+\frac{2 \epsilon}{1-\eta} .
\end{gathered}
$$

We will specify $\beta$ later. However, we note that it will be chosen in such a way that it guarantees $\eta<1 / 2$. Choose $b=1 / 2 \eta$. Then

$$
\left\|f_{m_{L}}\right\| \leqslant\left\|f_{B_{L-1}}\right\| 8 e^{-c_{1} \beta}+4 \epsilon .
$$

By (2.3) we get

$$
\left\|f_{\Gamma^{m_{L}}}\right\| \leqslant U\left(\left\|f_{m_{L}}\right\|+\epsilon\right) \leqslant U\left(\left\|f_{B_{L-1}}\right\| 8 e^{-c_{1} \beta}+5 \epsilon\right) .
$$

We note that in the proof of Theorem 2.5 we use the above inequality with $U=$ $V K^{r} \leqslant V K$. If $\left\|f_{B_{L-1}}\right\| \leqslant 10 U \epsilon$, then by (3.8),

$$
\left\|f_{m_{L}}\right\| \leqslant C U \epsilon .
$$

If $\left\|f_{B_{L-1}}\right\| \geqslant 10 U \epsilon$, then making $\beta$ sufficiently large to satisfy $16 U e^{-c_{1} \beta}<1$ so that $\beta=\left(C_{3} \ln (U+1)\right) / c_{1}$, we get

$$
U\left(\left\|f_{B_{L-1}}\right\| 8 e^{-c_{1} \beta}+5 \epsilon\right)<\left\|f_{B_{L-1}}\right\|
$$

and therefore

$$
\left\|f_{\Gamma^{m_{L}}}\right\|<\left\|f_{B_{L-1}}\right\|
$$

This implies that

$$
\left|\Gamma^{m_{L}}\right|<\left|\Gamma^{k}\right|-2^{L-2} .
$$

We begin with $f_{0}$ and apply the above argument (with $k=0$ ). As a result we either get the required inequality or we reduce the cardinality of support of $f$ from $|T|=K$ to $\left|\Gamma^{m_{L_{1}}}\right|<|T|-2^{L_{1}-2}, m_{L_{1}} \leqslant \beta 2^{a L_{1}}, a:=r q^{\prime}$. We continue the process and build a sequence $m_{L_{j}}$ such that $m_{L_{j}} \leqslant \beta 2^{a L_{j}}$ and after $m_{L_{j}}$ iterations 
we reduce the support by at least $2^{L_{j}-2}$. We also note that $m_{L_{j}} \leqslant \beta 2^{a} K^{a}$. We continue this process until the following inequality is satisfied for the first time:

$$
m_{L_{1}}+\cdots+m_{L_{n}} \geqslant 2^{2 a} \beta K^{a} .
$$

Then, clearly,

$$
m_{L_{1}}+\cdots+m_{L_{n}} \leqslant 2^{2 a+1} \beta K^{a} .
$$

Using the inequality

$$
\left(a_{1}+\cdots+a_{n}\right)^{\theta} \leqslant a_{1}^{\theta}+\cdots+a_{n}^{\theta}, \quad a_{j} \geqslant 0, \theta \in(0,1],
$$

we derive from (3.9),

$$
\begin{aligned}
2^{L_{1}-2}+\cdots+2^{L_{n}-2} & \geqslant\left(2^{a\left(L_{1}-2\right)}+\cdots+2^{a\left(L_{n}-2\right)}\right)^{\frac{1}{a}} \\
& \geqslant 2^{-2}\left(2^{a L_{1}}+\cdots+2^{a L_{n}}\right)^{\frac{1}{a}} \\
& \geqslant 2^{-2}\left((\beta)^{-1}\left(m_{L_{1}}+\cdots+m_{L_{n}}\right)\right)^{\frac{1}{a}} \geqslant K .
\end{aligned}
$$

Thus, after not more than $N:=2^{2 a+1} \beta K^{a}$ iterations we recover $f$ exactly and then $\left\|f_{N}\right\| \leqslant\left\|f_{0}-f\right\| \leqslant \epsilon$.

\section{Examples}

In this section we discuss applications of theorems from Section 2 for specific dictionaries $\mathcal{D}$. Mostly, $\mathcal{D}$ will be a basis $\Psi$ for $X$. Because of that we use $m$ instead of $K$ in the notation of sparse approximation. In some of our examples we take $X=L_{p}, 2 \leqslant p<\infty$. Then it is known that $\rho(u) \leqslant \gamma u^{2}$ with $\gamma=(p-1) / 2$. In some other examples we take $X=L_{p}, 1<p \leqslant 2$. Then it is known that $\rho(u) \leqslant \gamma u^{p}$, with $\gamma=1 / p$.

EXAMPLE 1 . Let $\Psi$ be a uniformly bounded orthogonal system normalized in $L_{p}(\Omega), 2 \leqslant p<\infty$, where $\Omega$ is a bounded domain. Then we have

$$
C_{1}(\Omega, p)\left\|\psi_{j}\right\|_{2} \leqslant\left\|\psi_{j}\right\|_{p} \leqslant C_{2}(\Omega, p)\left\|\psi_{j}\right\|_{2}, \quad j=1,2 \ldots
$$

Next, for $f=\sum_{i} c_{i}(f) \psi_{i}$,

$$
\begin{aligned}
\sum_{i \in A}\left|c_{i}(f)\right| & =\left\langle f, \sum_{i \in A}\left(\operatorname{sign} c_{i}(f)\right) \psi_{i}\left\|\psi_{i}\right\|_{2}^{-2}\right\rangle \\
& \leqslant\|f\|_{2}\left\|\sum_{i \in A}\left(\operatorname{sign} c_{i}(f)\right) \psi_{i}\right\| \psi_{i}\left\|_{2}^{-2}\right\|_{2} \\
& \leqslant C_{3}(\Omega, p)|A|^{1 / 2}\|f\|_{p} .
\end{aligned}
$$


Therefore $\Psi$ satisfies A3 with $D=\infty, V=C_{3}(\Omega, p), r=1 / 2$. Theorem 2.7 gives

$$
\left\|f_{C(t, p, D) m \ln (m+1)}\right\|_{p} \leqslant C \sigma_{m}\left(f_{0}, \Psi\right)_{p} .
$$

EXAMPLE 1q. Let $\Psi$ be a uniformly bounded orthogonal system normalized in $L_{p}(\Omega), 1<p \leqslant 2$, where $\Omega$ is a bounded domain. Then we have

$$
C_{1}(\Omega, p)\left\|\psi_{j}\right\|_{2} \leqslant\left\|\psi_{j}\right\|_{p} \leqslant C_{2}(\Omega, p)\left\|\psi_{j}\right\|_{2}, \quad j=1,2 \ldots
$$

Next, for $f=\sum_{i} c_{i}(f) \psi_{i}$,

$$
\begin{aligned}
\sum_{i \in A}\left|c_{i}(f)\right| & =\left\langle f, \sum_{i \in A}\left(\operatorname{sign} c_{i}(f)\right) \psi_{i}\left\|\psi_{i}\right\|_{2}^{-2}\right\rangle \\
& \leqslant\|f\|_{p}\left\|\sum_{i \in A}\left(\operatorname{sign} c_{i}(f)\right) \psi_{i}\right\| \psi_{i}\left\|_{2}^{-2}\right\|_{p^{\prime}} \\
& \leqslant C_{4}(\Omega, p)|A|^{1-1 / p^{\prime}}\|f\|_{p} .
\end{aligned}
$$

Therefore $\Psi$ satisfies A3 with $D=\infty, V=C_{4}(\Omega, p), r=1-1 / p^{\prime}$. Theorem 2.7 gives

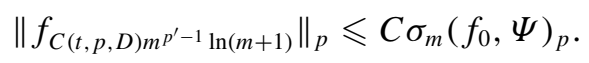

EXAMPLE 2. Let $\Psi$ be the real $d$-variate trigonometric system normalized in $L_{p}$, $2 \leqslant p<\infty$. Then Example 1 applies and gives for any $f_{0} \in L_{p}$,

$$
\left\|f_{C(t, p, d) m \ln (m+1)}\right\|_{p} \leqslant C \sigma_{m}\left(f_{0}, \Psi\right)_{p} .
$$

We note that (4.3) provides some progress in Open Problem 7.1 (p. 91) from [17].

EXAMPLE $2 \mathrm{q}$. Let $\Psi$ be the real $d$-variate trigonometric system normalized in $L_{p}, 1<p \leqslant 2$. Then Example $1 \mathrm{q}$ applies and gives for any $f_{0} \in L_{p}$,

$$
\left\|f_{C(t, p, d) m^{p^{\prime}-1} \ln (m+1)}\right\|_{p} \leqslant C \sigma_{m}\left(f_{0}, \Psi\right)_{p} .
$$

We need the concept of the cotype of a Banach space $X$. We say that $X$ has cotype $s$ if for any finite number of elements $u_{i} \in X$ we have the inequality

$$
\left(\text { Average }_{ \pm}\left\|\sum_{i} \pm u_{i}\right\|^{s}\right)^{1 / s} \geqslant C_{s}\left(\sum_{i}\left\|u_{i}\right\|^{s}\right)^{1 / s} .
$$

It is known that the $L_{p}$ spaces with $2 \leqslant p<\infty$ have cotype $s=p$ and $L_{p}$ spaces with $1<p \leqslant 2$ have cotype two. 
REMARK 4.1. Suppose $\mathcal{D}$ is $(K, K)$-unconditional with a constant $U$. Assume that $X$ is of cotype $s$ with a constant $C_{s}$. Then $\mathcal{D}$ has the Nikol'skii-type $\ell_{1} X$ property with parameters $K, 1-1 / s$ and $C_{1}=2 U C_{s}^{-1}$.

Proof. Our assumption about $(K, K)$-unconditionality implies: for any $A,|A| \leqslant$ $K$, we have

$$
\left\|\sum_{i \in A} \pm x_{i} g_{i}\right\| \leqslant 2 U\left\|\sum_{i \in A} x_{i} g_{i}\right\| .
$$

Therefore, by the $s$-cotype assumption,

$$
\left\|\sum_{i \in A} x_{i} g_{i}\right\|^{s} \geqslant(2 U)^{-s} C_{s}^{s} \sum_{i \in A}\left|x_{i}\right|^{s} .
$$

This implies

$$
\sum_{i \in A}\left|x_{i}\right| \leqslant|A|^{1-1 / s}\left(\sum_{i \in A}\left|x_{i}\right|^{s}\right)^{1 / s} \leqslant 2 U C_{s}^{-1}|A|^{1-1 / s}\left\|\sum_{i \in A} x_{i} g_{i}\right\| .
$$

EXAMPLE 3. Let $X$ be a Banach space with $\rho(u) \leqslant \gamma u^{q}, 1<q \leqslant 2$, and with cotype $s$. Let $\Psi$ be an unconditional basis for $X$ normalized in $X$. Then $U \leqslant C(X$, $\Psi)$ and $\Psi$ satisfies $\mathbf{A 2}$ with $D=\infty$ and any $K$.

By Remark $4.1 \Psi$ satisfies A1 with $r=1-1 / s$. Theorem 2.4 gives

$$
\left\|f_{C(t, X, \Psi) m^{(1-1 / s) q^{\prime}}}\right\| \leqslant C \sigma_{m}\left(f_{0}, \Psi\right) .
$$

EXAmPLE 4. Let $\Psi$ be the multivariate Haar basis $\mathcal{H}_{p}^{d}=\mathcal{H}_{p} \times \cdots \times \mathcal{H}_{p}$ normalized in $L_{p}, 2 \leqslant p<\infty$. It is an unconditional basis. Also it is known that an $L_{p}$ space with $2 \leqslant p<\infty$ has cotype $s=1 / p$. Therefore, Example 3 applies in this case. We give a direct argument here. It is an unconditional basis and therefore $U \leqslant C(p, d)$. Next, for any $A$,

$$
\left\|\sum_{i \in A} x_{i} H_{i, p}\right\|_{p} \geqslant C(p, d)\left(\sum_{i \in A}\left|x_{i}\right|^{p}\right)^{1 / p} \geqslant C(p, d)|A|^{\frac{1}{p}-1} \sum_{i \in A}\left|x_{i}\right| .
$$

Therefore, we can take $r=1 / p^{\prime}$. Theorem 2.4 gives

$$
\left\|f_{C(t, p, d) m^{2 / p^{\prime}}}\right\|_{p} \leqslant C \sigma_{m}\left(f_{0}, \mathcal{H}_{p}^{d}\right)_{p} .
$$

Inequality (4.6) provides some progress in Open Problem 7.2 (p. 91) from [17] for the case $2<p<\infty$. 
EXAMPLE 4q. Let $\Psi$ be the univariate Haar basis $\mathcal{H}_{p}=\left\{H_{I, p}\right\}_{I}$ normalized in $L_{p}, 1<p \leqslant 2$, where the $H_{I, p}$ are the Haar functions indexed by dyadic intervals of support of $H_{I, p}$ (we index function 1 by $[0,1]$ and the first Haar function by $(0$, 1]). Then for any finite set $A$ of dyadic intervals we have for $f=\sum_{I} c_{I}(f) H_{I, p}$,

$$
\sum_{I \in A}\left|c_{I}\right|=\left\langle f, f_{A}^{*}\right\rangle, \quad f_{A}^{*}:=\sum_{I \in A}\left(\operatorname{sign} c_{I}(f)\right) H_{I, p}\left\|H_{I, p}\right\|_{2}^{-2} .
$$

Therefore,

$$
\sum_{I \in A}\left|c_{I}\right| \leqslant\|f\|_{p}\left\|f_{A}^{*}\right\|_{p^{\prime}}
$$

It is easy to check that

$$
\left\|H_{I, p}\right\|_{p^{\prime}}\left\|H_{I, p}\right\|_{2}^{-2}=|I|^{-1 / p}|I|^{1 / p^{\prime}}|I|^{-(1-2 / p)}=1 .
$$

By Lemma 1.23, p. 28, from [18] we get

$$
\left\|f_{A}^{*}\right\|_{p^{\prime}} \leqslant C(p)|A|^{1 / p^{\prime}} .
$$

Thus

$$
\sum_{I \in A}\left|c_{I}\right| \leqslant C(p)|A|^{1 / p^{\prime}}\|f\|_{p}
$$

This means that $\mathcal{H}_{p}$ satisfies $\mathbf{A 3}$ with $V=C(p)$ and $r=1 / p^{\prime}$. Also it is an unconditional basis and therefore satisfies $\mathbf{A 2}$ with $U=C(p)$. It is known that an $L_{p}$ space with $1<p \leqslant 2$ has modulus of smoothness $\rho(u) \leqslant \gamma u^{p}$. Therefore, Theorem 2.8 applies in this case and gives

$$
\left\|f_{C(t, p) m}\right\|_{p} \leqslant C \sigma_{m}\left(f_{0}, \mathcal{H}_{p}\right)_{p} .
$$

Inequality (4.7) solves the Open Problem 7.2 (p. 91) from [17] in the case $1<$ $p \leqslant 2$.

EXAMPLE 5. Let $X$ be a Banach space with $\rho(u) \leqslant \gamma u^{2}$. Assume that $\Psi$ is a normalized Schauder basis for $X$. Then for any $f=\sum_{i} c_{i}(f) \psi_{i}$,

$$
\sum_{i \in A}\left|c_{i}(f)\right| \leqslant C(\Psi)|A|\|f\| .
$$

This implies that $\Psi$ satisfies $\mathbf{A 3}$ with $D=\infty, V=C(\Psi), r=1$ and any $K$. Theorem 2.7 gives

$$
\left\|f_{C(t, X, \Psi) m^{2} \ln m}\right\| \leqslant C \sigma_{m}\left(f_{0}, \Psi\right) .
$$


We note that the above simple argument still works if we replace the assumption that $\Psi$ is a Schauder basis by the assumption that a dictionary $\mathcal{D}$ is $(1, D)$ unconditional with constant $U$. Then we obtain

$$
\left\|f_{C(t, \gamma, U) K^{2} \ln K}\right\| \leqslant C \sigma_{K}\left(f_{0}, \Psi\right), \quad \text { for } K+C(t, \gamma, U) K^{2} \ln K \leqslant D .
$$

EXAMPlE 5q. Let $X$ be a Banach space with $\rho(u) \leqslant \gamma u^{q}, 1<q \leqslant 2$. Assume that $\Psi$ is a normalized Schauder basis for $X$. Then for any $f=\sum_{i} c_{i}(f) \psi_{i}$,

$$
\sum_{i \in A}\left|c_{i}(f)\right| \leqslant C(\Psi)|A|\|f\| .
$$

This implies that $\Psi$ satisfies $\mathbf{A 3}$ with $D=\infty, V=C(\Psi), r=1$ and any $T$. Theorem 2.7 gives

$$
\left\|f_{C(t, X, \Psi) m q^{\prime} \ln m}\right\| \leqslant C \sigma_{m}\left(f_{0}, \Psi\right) .
$$

We note that the above simple argument still works if we replace the assumption that $\Psi$ is a Schauder basis by the assumption that a dictionary $\mathcal{D}$ is $(1, D)$ unconditional with constant $U$. Then we obtain

$$
\left\|f_{C(t, \gamma, q, U) K^{q^{\prime}} \ln K}\right\| \leqslant C \sigma_{K}\left(f_{0}, \mathcal{D}\right), \quad \text { for } K+C(t, \gamma, q, U) K^{q^{\prime}} \ln K \leqslant D .
$$

We now discuss an application of Theorem 2.1 to quasigreedy bases. We begin with a brief introduction to the theory of quasigreedy bases. Let $X$ be an infinitedimensional separable Banach space with a norm $\|\cdot\|:=\|\cdot\|_{X}$ and let $\Psi:=$ $\left\{\psi_{m}\right\}_{m=1}^{\infty}$ be a normalized basis for $X$. The concept of a quasigreedy basis was introduced in [8].

DEFINITION 4.1. The basis $\Psi$ is called quasigreedy if there exists some constant $C$ such that

$$
\sup _{m}\left\|G_{m}(f, \Psi)\right\| \leqslant C\|f\| \text {. }
$$

Subsequently, Wojtaszczyk [23] proved that these are precisely the bases for which the TGA merely converges, that is,

$$
\lim _{n \rightarrow \infty} G_{n}(f)=f
$$

The following lemma is from [3] (see also [5]).

LEMMA 4.1. Let $\Psi$ be a quasigreedy basis of $X$. Then for any finite set of indices $\Lambda$ we have for all $f \in X$,

$$
\left\|S_{\Lambda}(f, \Psi)\right\| \leqslant C \ln (|\Lambda|+1)\|f\| .
$$


We now formulate a result concerning quasigreedy bases in $L_{p}$ spaces. The following theorem is from [19]. We note that for the case $p=2$, Theorem 4.1 was proved in [23]. Some notation first. For a given element $f \in X$ we consider the expansion

$$
f=\sum_{k=1}^{\infty} c_{k}(f) \psi_{k}
$$

and the decreasing rearrangement of its coefficients

$$
\left|c_{k_{1}}(f)\right| \geqslant\left|c_{k_{2}}(f)\right| \geqslant \cdots \text {. }
$$

Define

$$
a_{n}(f):=\left|c_{k_{n}}(f)\right|
$$

THEOREM 4.1. Let $\Psi=\left\{\psi_{m}\right\}_{m=1}^{\infty}$ be a quasigreedy basis of the $L_{p}$ space, $1<$ $p<\infty$. Then for each $f \in X$ we have

$$
\begin{array}{ll}
C_{1}(p) \sup _{n} n^{1 / p} a_{n}(f) \leqslant\|f\|_{p} \leqslant C_{2}(p) \sum_{n=1}^{\infty} n^{-1 / 2} a_{n}(f), & 2 \leqslant p<\infty ; \\
C_{3}(p) \sup _{n} n^{1 / 2} a_{n}(f) \leqslant\|f\|_{p} \leqslant C_{4}(p) \sum_{n=1}^{\infty} n^{1 / p-1} a_{n}(f), & 1<p \leqslant 2 .
\end{array}
$$

EXAMPLE 6 . Let $\Psi$ be a normalized quasigreedy basis for $L_{p}, 2 \leqslant p<\infty$. Theorem 4.1 implies for any $f=\sum_{i} c_{i}(f) \psi_{i}$,

$$
\sum_{i \in A}\left|c_{i}(f)\right| \leqslant \sum_{n=1}^{|A|} a_{n}(f) \leqslant C_{1}(p)^{-1} \sum_{n=1}^{|A|} n^{-1 / p}\|f\|_{p} \leqslant C(p)|A|^{1-1 / p}\|f\|_{p} .
$$

This means that $\Psi$ satisfies $\mathbf{A 3}$ with $D=\infty, V=C(p), r=1-1 / p$. Theorem 2.7 gives

$$
\left\|f_{C(t, p) m^{2(1-1 / p)} \ln (m+1)}\right\| \leqslant C \sigma_{m}\left(f_{0}, \Psi\right) .
$$

EXAMPLE 6q. Let $\Psi$ be a normalized quasigreedy basis for $L_{p}, 1<p \leqslant 2$. Theorem 4.1 implies for any $f=\sum_{i} c_{i}(f) \psi_{i}$,

$$
\sum_{i \in A}\left|c_{i}(f)\right| \leqslant \sum_{n=1}^{|A|} a_{n}(f) \leqslant C_{3}(p)^{-1} \sum_{n=1}^{|A|} n^{-1 / 2}\|f\|_{p} \leqslant C(p)|A|^{1 / 2}\|f\|_{p} .
$$

This means that $\Psi$ satisfies A3 with $D=\infty, V=C(p), r=1 / 2$. Theorem 2.7 gives

$$
\left\|f_{C(t, p) m^{p^{\prime} / 2} \ln (m+1)}\right\| \leqslant C \sigma_{m}\left(f_{0}, \Psi\right) .
$$


EXAMPLE 7. Let $\Psi$ be a normalized uniformly bounded orthogonal quasigreedy basis for $L_{p}, 2 \leqslant p<\infty$. For the existence of such bases see [13]. Then orthogonality implies that we can take $r=1 / 2$. We obtain from Lemma 4.1 that $\Psi$ is $(K, \infty)$ unconditional with $U \leqslant C \ln (K+1)$. Theorem 2.8 gives

$$
\left\|f_{C(t, p, \Psi) m \ln \ln (m+3)}\right\|_{p} \leqslant C \sigma_{m}\left(f_{0}, \Psi\right)_{p} .
$$

EXAMPLE 7q. Let $\Psi$ be a normalized uniformly bounded orthogonal quasigreedy basis for $L_{p}, 1<p \leqslant 2$. For the existence of such bases see [13]. Then orthogonality implies that we can take $r=1 / 2$. We obtain from Lemma 4.1 that $\Psi$ is $(K, \infty)$ unconditional with $U \leqslant C \ln (K+1)$. Theorem 2.8 gives

$$
\left\|f_{C(t, p, \Psi) m^{p^{\prime} / 2} \ln \ln (m+3)}\right\|_{p} \leqslant C \sigma_{m}\left(f_{0}, \Psi\right)_{p} .
$$

\section{Discussion}

We study sparse approximation. In a general setting we study an algorithm (approximation method) $\mathcal{A}=\left\{A_{m}(\cdot, \mathcal{D})\right\}_{m=1}^{\infty}$ with respect to a given dictionary $\mathcal{D}$. The sequence of mappings $A_{m}(\cdot, \mathcal{D})$ defined on $X$ satisfies the following condition: for any $f \in X, A_{m}(f, \mathcal{D}) \in \Sigma_{m}(\mathcal{D})$. In other words, $A_{m}$ provides an $m$-term approximant with respect to $\mathcal{D}$. It is clear that for any $f \in X$ and any $m$ we have

$$
\left\|f-A_{m}(f, \mathcal{D})\right\| \geqslant \sigma_{m}(f, \mathcal{D}) .
$$

We are interested in such pairs $(\mathcal{D}, \mathcal{A})$ for which the algorithm $\mathcal{A}$ provides approximation close to the best $m$-term approximation. We introduce the corresponding definitions.

Definition 5.1. We say that $\mathcal{D}$ is a greedy dictionary with respect to $\mathcal{A}$ if there exists a constant $C_{0}$ such that for any $f \in X$ we have

$$
\left\|f-A_{m}(f, \mathcal{D})\right\| \leqslant C_{0} \sigma_{m}(f, \mathcal{D}) .
$$

If $\mathcal{D}$ is a greedy dictionary with respect to $\mathcal{A}$ then $\mathcal{A}$ provides ideal (up to a constant $C_{0}$ ) $m$-term approximations for every $f \in X$.

Definition 5.2. We say that $\mathcal{D}$ is an almost greedy dictionary with respect to $\mathcal{A}$ if there exist two constants $C_{1}$ and $C_{2}$ such that for any $f \in X$ we have

$$
\left\|f-A_{C_{1} m}(f, \mathcal{D})\right\| \leqslant C_{2} \sigma_{m}(f, \mathcal{D}) .
$$


If $\mathcal{D}$ is an almost greedy dictionary with respect to $\mathcal{A}$ then $\mathcal{A}$ provides almost ideal sparse approximation. It provides a $C_{1} m$-term approximant as good (up to a constant $C_{2}$ ) as the ideal $m$-term approximant for every $f \in X$. We also need a more general definition. Let $\phi(u)$ be a function such that $\phi(u) \geqslant 1$.

Definition 5.3. We say that $\mathcal{D}$ is a $\phi$-greedy dictionary with respect to $\mathcal{A}$ if there exists a constant $C_{3}$ such that for any $f \in X$ we have

$$
\left\|f-A_{\phi(m) m}(f, \mathcal{D})\right\| \leqslant C_{3} \sigma_{m}(f, \mathcal{D}) .
$$

If $\mathcal{D}=\Psi$ is a basis then in the above definitions we replace dictionary by basis. For the case where $\mathcal{A}=\left\{G_{m}(\cdot, \Psi)\right\}_{m=1}^{\infty}$ is the TGA, the theory of greedy and almost greedy bases is well developed (see [18]). We present two results on the characterization of these bases. A basis $\Psi$ in a Banach space $X$ is called democratic if there is a constant $C(\Psi)$ such that

$$
\left\|\sum_{k \in A} \psi_{k}\right\| \leqslant C(\Psi)\left\|\sum_{k \in B} \psi_{k}\right\|
$$

if $|A|=|B|$. This concept was introduced in [8]. In [4] we defined a democratic basis as the one satisfying (5.4) if $|A| \leqslant|B|$. It is known that for quasigreedy bases the above two definitions are equivalent. It was proved in [8] (see Theorem 1.15, p. 18, [18]) that a basis is greedy with respect to the TGA if and only if it is unconditional and democratic. It was proved in [4] (see Theorem 1.37, p. 38, [18]) that a basis is almost greedy with respect to the TGA if and only if it is quasigreedy and democratic.

Example $4 \mathrm{q}$ is the first result concerning almost greedy bases with respect to the WCGA in Banach spaces. It shows that the univariate Haar basis is an almost greedy basis with respect to the WCGA in the $L_{p}$ spaces for $1<p \leqslant 2$. Example 1 shows that uniformly bounded orthogonal bases are $\phi$-greedy bases with respect to the WCGA with $\phi(u)=C(t, p, D) \ln (u+1)$ in the $L_{p}$ spaces for $2 \leqslant p<$ $\infty$. We do not know whether these bases are almost greedy with respect to the WCGA. They are good candidates for that.

It is known (see [18], p. 17) that the univariate Haar basis is a greedy basis with respect to the TGA for all $L_{p}, 1<p<\infty$. Example 4 only shows that it is a $\phi$-greedy basis with respect to the WCGA with $\phi(u)=C(t, p) u^{1-2 / p}$ in the $L_{p}$ spaces for $2 \leqslant p<\infty$. It is much weaker than the corresponding results for the $\mathcal{H}_{p}, 1<p \leqslant 2$, and for the trigonometric system, $2 \leqslant p<\infty$ (see Example 2). We do not know whether this result on the Haar basis can be substantially improved. At the level of our today's technique we can observe that the Haar basis is ideal (a greedy basis) for the TGA in $L_{p}, 1<p<\infty$, almost 
ideal (an almost greedy basis) for the WCGA in $L_{p}, 1<p \leqslant 2$, and that the trigonometric system is very good for the WCGA in $L_{p}, 2 \leqslant p<\infty$.

Example 2q shows that our results for the trigonometric system in $L_{p}, 1<p<$ 2 , are not as strong as for $2 \leqslant p<\infty$. We do not know whether this is because of the lack of an appropriate technique or whether it reflects the nature of the WCGA with respect to the trigonometric system.

We note that the properties of a given basis with respect to the TGA and WCGA could be very different. For instance, the class of quasigreedy bases (with respect to the TGA) is a rather narrow subset of all bases. It is close in a certain sense to the set of unconditional bases. The situation is completely different for the WCGA. If $X$ is uniformly smooth, then the WCGA converges for each $f \in X$ with respect to any dictionary in $X$. Moreover, Example 5q shows that if $X$ is a Banach space with $\rho(u) \leqslant \gamma u^{q}$, then any basis $\Psi$ is $\phi$-greedy with respect to the WCGA with $\phi(u)=C(t, X, \Psi) u^{q^{\prime}-1} \ln (u+1)$.

It is interesting to compare Theorem 2.3 with the following known result. The following theorem provides a rate of convergence (see [18], p. 347). As above, we denote by $A_{1}(\mathcal{D})$ the closure in $X$ of the convex hull of the symmetrized dictionary $\mathcal{D}^{ \pm}:=\{ \pm g: g \in \mathcal{D}\}$.

THEOREM 5.1. Let $X$ be a uniformly smooth Banach space with modulus of smoothness $\rho(u) \leqslant \gamma u^{q}, 1<q \leqslant 2$. Take a number $\epsilon \geqslant 0$ and two elements $f_{0}, f^{\epsilon}$ from $X$ such that

$$
\left\|f_{0}-f^{\epsilon}\right\| \leqslant \epsilon, \quad f^{\epsilon} / A(\epsilon) \in A_{1}(\mathcal{D}),
$$

with some number $A(\epsilon)>0$. Then, for the WCGA we have

$$
\left\|f_{m}^{c, \tau}\right\| \leqslant \max \left(2 \epsilon, C(q, \gamma)(A(\epsilon)+\epsilon)\left(1+\sum_{k=1}^{m} t_{k}^{q^{\prime}}\right)^{-1 / q^{\prime}}\right) .
$$

Both Theorem 5.1 and Theorem 2.3 provide stability of the WCGA with respect to noise. In order to apply them for noisy data we interpret $f_{0}$ as a noisy version of a signal and $f^{\epsilon}$ as a noiseless version of a signal. Then, the assumption $f^{\epsilon} / A(\epsilon) \in A_{1}(\mathcal{D})$ describes our smoothness assumption on the noiseless signal and the assumption $f^{\epsilon} \in \Sigma_{K}(\mathcal{D})$ describes our structural assumption on the noiseless signal. In fact, Theorem 5.1 simultaneously takes care of two issues: noisy data and approximation in an interpolation space. Theorem 5.1 can be applied for the approximation of $f_{0}$ under the assumption that $f_{0}$ belongs to one of the interpolation spaces between $X$ and the space generated by the $A_{1}(\mathcal{D})$-norm (atomic norm). 
We now give some historical remarks on the Lebesgue-type inequalities for redundant dictionaries. By a Lebesgue-type inequality we mean an inequality that provides an upper bound for the error of a particular method of approximation of $f$ by elements of a special form, say, form $\mathcal{A}$, as the best-possible approximation of $f$ by elements of the form $\mathcal{A}$. In our paper the method of approximation is the WCGA which provides an $m$-term approximant after the $m$ th iteration. Thus, form $\mathcal{A}$ is a linear combination of at most $m$ dictionary elements. Therefore, we compare the error of the WCGA after $m$ iterations with the best $m$-term approximation. First Lebesgue-type inequalities for redundant dictionaries were proved for the orthogonal matching pursuit (OMP), which is the WCGA in a Hilbert space with the weakness parameter $t=1$, under the assumption of incoherence on the dictionary.

Define

$$
M(\mathcal{D}):=\sup _{g \neq h ; g, h \in \mathcal{D}}|\langle g, h\rangle|,
$$

the coherence parameter of a dictionary $\mathcal{D}$. The first general Lebesgue-type inequality for the OMP for the $M$-coherent dictionary was obtained in [6]. The authors proved that for the residual $f_{m}^{o}$ of the OMP after $m$ iterations one has

$$
\left\|f_{m}^{o}\right\| \leqslant 8 m^{1 / 2} \sigma_{m}(f) \quad \text { for } m<1 /(32 M) .
$$

The constants in this inequality were improved in [21]:

$$
\left\|f_{m}^{o}\right\| \leqslant(1+6 m)^{1 / 2} \sigma_{m}(f) \text { for } m<1 /(3 M) .
$$

Further results were obtained in [2]: assume that $m \leqslant 0.05 M^{-2 / 3}$; then we have

$$
\left\|f_{[m \log m]}^{o}\right\| \leqslant 24 \sigma_{m}(f) .
$$

The following inequality was obtained in [20]. For any $\delta \in(0,1 / 4]$, set $L(\delta):=$ $[1 / \delta]+1$. Assume that $m$ is such that $20 M m^{1+\delta} 2^{L(\delta)} \leqslant 1$. Then we have

$$
\left\|f_{m\left(2^{L(\delta)+1}-1\right)}^{o}\right\| \leqslant \sqrt{3} \sigma_{m}(f) .
$$

Recently, the above Lebesgue-type inequality was improved in [9]:

$$
\left\|f_{2 m}^{o}\right\| \leqslant 3 \sigma_{m}(f)
$$

for $m \leqslant(20 M)^{-1}$.

The assumption of incoherence on a dictionary is stronger than the restricted isometry property (RIP) assumption. The corresponding Lebesgue-type inequalities for the OMP under the RIP assumption were not known for a while. As a result, new greedy-type algorithms were introduced, with the proof 
of exact recovery of sparse signals and the Lebesgue-type inequalities for these algorithms: the regularized orthogonal matching pursuit (see [12]), the compressive sampling matching pursuit (CoSaMP; see [11]), and the subspace pursuit (SP; see [1]). The OMP is simpler than the CoSaMP and the SP; however, at the time of invention of the CoSaMP and the SP these algorithms provided exact recovery of sparse signals and the Lebesgue-type inequalities for dictionaries satisfying the restricted isometry property (RIP) (see [11] and [1]). The corresponding results for the OMP were not known at that time. Later, a breakthrough result in this direction was obtained by Zhang [24]. In particular, he proved that if $\mathcal{D} \in R(31 K, \delta), \delta<1 / 3$, then the OMP recovers exactly all $K$-sparse signals within $30 K$ iterations. In other words, $f_{30 K}^{o}=0$. It would be interesting and difficult to improve the constant 30 to the optimal one. There are several papers devoted to this problem (see [7], [22], and [10]). In this paper we developed Zhang's technique to obtain recovery results and the Lebesgue-type inequalities in the Banach space setting.

Concluding, we briefly describe the contribution of this paper. First, we present a study of the Lebesque-type inequalities with respect to the WCGA in Banach spaces with $\rho(u) \leqslant \gamma u^{q}, 1<q \leqslant 2$, under conditions A1 and A2. For the case $q=2$ this has been done in [10]. The case $1<q<2$ uses the same ideas as [10]. Second, we introduce a new condition $\mathbf{A 3}$ and study the WCGA with respect to dictionaries satisfying either $\mathbf{A} 3$ or $\mathbf{A 2}$ and A3. Condition $\mathbf{A 3}$ and a combination of $\mathbf{A} 2$ and $\mathbf{A} 3$ turn out to be more powerful in applications than A1 combined with A2. Third, we apply the general theory developed in Sections 2 and 3 for bases. Surprisingly, this technique works very well for very different bases. It provides first results on the Lebesque-type inequalities for the WCGA with respect to bases in Banach spaces. Some of these results (for the $\mathcal{H}_{p}, 1<p \leqslant 2$, and for the $\left.\mathcal{R} \mathcal{T}_{p}, 2 \leqslant p<\infty\right)$ are strong. This demonstrates that the technique used is an appropriate and powerful method.

\section{Acknowledgements}

This research was supported by NSF grant DMS-1160841.

\section{References}

[1] W. Dai and O. Milenkovic, 'Subspace pursuit for compressive sensing signal reconstruction', IEEE Trans. Inform. Theory 55 (2009), 2230-2249.

[2] D. Donoho, M. Elad and V. N. Temlyakov, 'On the Lebesgue type inequalities for greedy approximation', J. Approx. Theory 147 (2007), 185-195.

[3] S.J. Dilworth, N.J. Kalton and D. Kutzarova, 'On the existence of almost greedy bases in Banach spaces', Studia Math. 158 (2003), 67-101. 
[4] S. J. Dilworth, N. J. Kalton, D. Kutzarova and V. N. Temlyakov, 'The thresholding greedy algorithm, greedy bases, and duality', Constr. Approx. 19 (2003), 575-597.

[5] S. J. Dilworth, M. Soto-Bajo and V. N. Temlyakov, 'Quasi-greedy bases and Lebesgue-type inequalities', Sud. Math. 211 (2012), 41-69.

[6] A. C. Gilbert, S. Muthukrishnan and M. J. Strauss, 'Approximation of functions over redundant dictionaries using coherence', The 14th Annual ACM-SIAM Symposium on Discrete Algorithms (2003).

[7] S. Foucart, 'Sparse recovery algorithms: sufficient conditions in terms of restricted isometry constants', in: Approximation Theory XIII: San Antonio, 2010 (2012), 65-77.

[8] S. V. Konyagin and V. N. Temlyakov, 'A remark on greedy approximation in Banach spaces', East. J. Approx. 5 (1999), 365-379.

[9] E. D. Livshitz, 'On the optimality of the Orthogonal Greedy Algorithm for $\mu$-coherent dictionaries', J. Approx. Theory 164 (5) (2012), 668-681.

[10] E. D. Livshitz and V. N. Temlyakov, 'Sparse approximation and recovery by greedy algorithms', arXiv:1303.3595v1 [math.NA] 14 Mar 2013.

[11] D. Needell and J. A. Tropp, 'CoSaMP: iterative signal recovery from incomplete and inaccurate samples', Appl. Comput. Harmonic Anal. 26 (2009), 301-321.

[12] D. Needell and R. Vershynin, 'Uniform uncertainty principle and signal recovery via orthogonal matching pursuit', Found. Comput. Math. 9 (2009), 317-334.

[13] M. Nielsen, 'An example of an almost greedy uniformly bounded orthonormal basis for $L_{p}(0,1)$ ', J. Approx. Theory 149 (2007), 188-192.

[14] D. Savu and V. N. Temlyakov, 'Lebesgue-type inequalities for greedy approximation in Banach spaces', IEEE Trans. Inform. Theory 58 (2013), 1098-1106.

[15] V. N. Temlyakov, 'Greedy algorithm and $m$-term trigonometric approximation', Constr. Approx. 14 (1998), 569-587.

[16] V. N. Temlyakov, 'Greedy algorithms in Banach spaces', Adv. Comput. Math. 14 (2001), 277-292.

[17] V. N. Temlyakov, 'Nonlinear methods of approximation', Found. Comput. Math. 3 (2003), 33-107.

[18] V. N. Temlyakov, Greedy Approximation, (Cambridge University Press, 2011).

[19] V. N. Temlyakov, M. Yang and P. Ye, 'Greedy approximation with regard to non-greedy bases', Adv. Comput. Math. 34 (2011), 319-337.

[20] V. Temlyakov and P. Zheltov, 'On performance of greedy algorithms', J. Approx. Theory 163 (2011), 1134-1145.

[21] J. A. Tropp, 'Greed is good: Algorithmic results for sparse approximation', IEEE Trans. Inform. Theory 50 (2004), 2231-2242.

[22] J. Wang and B. Shim, 'Improved recovery bounds of orthogonal matching pursuit using restricted isometry property', arXiv:1211.4293v1 [cs.IT] 19 Nov 2012.

[23] P. Wojtaszczyk, 'Greedy algorithm for general biorthogonal systems', J. Approx. Theory 107 (2000), 293-314.

[24] T. Zhang, 'Sparse recovery with orthogonal matching pursuit under RIP', IEEE Trans. Inform. Theory 57 (2011), 6215-6221. 